\title{
Technology-Assisted Carpal Tunnel Syndrome Rehabilitation using Serious Games: The Roller Ball Example
}

\author{
Loannis Pachoulakis and Diana Tsilidi \\ Department of Informatics Engineering, Technological Educational Institute of Crete, \\ Heraklion-Crete, Greece; \\ ip@ie.teicrete.gr; dtsilidi@gmail.com
}

\begin{abstract}
The adverse effects of improper posture and unhealthy computer usage habits on human physiology have been well documented. Prominent among these, the Carpal Tunnel Syndrome (CTS) is a medical condition where the median nerve passing through the wrist's carpal tunnel is compressed causing pain, numbness and tingling in affected parts of the hand. Depending on the severity of the symptoms, treatment may include physiotherapy and/or surgery. The present paper discusses possibilities that technology-driven physiotherapy based on serious games can augment a more traditional physiotherapy exercise curriculum. Accordingly, we present a Unity3D game called Roller Ball, the scenario of which combines CTS-specific physiotherapy exercises in a natural scenario-based way to guide a ball across a bridge in a 3D scene. The game employs the Leap Motion sensor, whose detailed wrist and hand (including fingers) tracking abilities make it a promising hardware platform for rehabilitation oriented exercises intended for patients suffering from CTS.
\end{abstract}

Keywords: Carpal Tunnel Syndrome, Physical Therapy, Leap Motion, Sensors, Serious Games.

\section{Introduction}

Carpal tunnel syndrome (CTS) is a medical condition in which the median nerve is compressed as it travels through the wrist's carpal tunnel, causing pain, numbness and tingling in parts of the hand that receive sensation from the median nerve [1]. Many patients resort to physiotherapy to reduce the pain and follow an exercise schedule for mobility and strength. Conservative management of symptoms related to CTS include exercises which involve tendon gliding of the finger flexor tendons and nerve gliding of the median nerve [2], [3]. Individualized therapy may also include additional exercises to increase muscle strength in the hand, fingers and forearm - and in some cases, the trunk and postural back muscles - as well as stretching exercises to improve flexibility in the wrist, hand and fingers. The goal of physical therapy is to reduce the severity of symptoms, to possibly eliminate the need for surgery and to permit the patient to be active and functional in everyday life. Physical therapy is also important following CTS surgery to help patients restore strength to the wrist and to retrain them to stray from the bad habits that may have led to the symptoms in the first place [4]. However, physical therapy is a slow process and it often takes weeks or months before positive effects are felt. During therapy, patients have to perform time consuming, repetitive and, as a result, boring exercises. Whereas in some cases the presence of a physical therapist may not be strictly necessary after the first few establishing sessions, in other cases mounting physiotherapy expenses may make it difficult to schedule sessions as frequently as may be required. It is fairly common for patients to attend a number of intensive physiotherapy sessions and then be given an exercise curriculum and schedule to 
follow at home on a regular basis, checking with the physiotherapist more sparingly. As lack of motivation arising from the repeatability and predictability of the exercises sets in, patients begin to neglect to perform their exercise program at home as regularly as they should [5]. Therefore, transforming traditional physiotherapy exercises into interesting and hopefully fun games carries a special promise to unsupervised therapy, as goal oriented, highly interactive games can provide the necessary motivation to carry out and complete an exercise schedule at home. In addition, game features such as being in control of a 3D world, combined with elements such as achievements, high scores, rewards and positive feedback, can draw patients to lengthier game sessions and thus speed up their recovery [6].

The effectiveness of a therapeutic game system is commonly based on assessing patient progress during rehabilitation. Sensor technology has matured sufficiently to persuasively and un-obstructively be integrated in game solutions. Indeed, three ubiquitous motion tracking sensors in today's games (Nintendo's Wii, Microsoft's Kinect and the Leap Motion sensor) have different philosophies and follow different approaches to bring motion capture to their host game platforms. Nintendo's Wii, released in 2006, was the first home video game console to use motion-sensing technology [7]. The primary Wii remote controller detects movement in three dimensions. In addition to the usual buttons, it has a built-in accelerometer combined with infrared camera which allows it to sense its position in 3D space and detect acceleration along three axes. The remote controller connects to Wii using Bluetooth with an approximate range of 9 meters. Additional Wii-compatible controllers include the Nunchuck (which features an accelerometer and a traditional analog stick with trigger buttons) as well as a Balance Board which contains several sensors that calculate the mass of the player standing on it and his/her center of gravity. There have been attempts to combine the benefits of Nintendo's Wii with other projects to produce new integrated rehabilitation systems [8].

Wii has a large user base and has been effective in exercising the entire human body. For example, post-stroke patient condition has shown to improve after the use of Wii Fit Balance Board for rehabilitation. Whereas many Wii-based games make use of the Balance Board extension, it is a risky piece of hardware for Parkinson's disease patients, as it may lead to falls [9]. In addition, all Wii games require a handheld controller to capture the player's movement, which may be troublesome for some patients. In addition, excessive game play, tiredness and the enthusiasm of group play may lead to injuries related to falls or player body part collisions. Cases reported include acute tendonitis of right infraspinatus, dislocation of left patella, medial meniscal tear, and acute onset of carpel tunnel syndrome (see for example [10], also [11]). Finally, Wii's remote control is supposed to be grasped and does not allow delicate hand movements (flexions, extensions, deviations).

Microsoft's Kinect is a motion sensor bar initially built as an alternative input device to a remote control to the Xbox 360 video game console and later also released for Windows PCs. Kinect uses a Natural User Interface (NUI) to enable user interaction with the game environment through gestures or voice commands. The sensor bar contains an RGB camera and a depth sensor composed of an infrared emitter and a monochrome CMOS to offer robust 3D data capturing as well as a multi-array microphone used to isolate players' voices from the noise in the room. The supporting Software Development Kit (SDK) enables recognition of six players and can provide continuous skeletal tracking of two of them and can be programmed in $\mathrm{C}++, \mathrm{C \#}$, and Visual Basic.Net [12].

Evaluation of the Kinect sensor in medical applications requiring a motion capture device show that it is capable of sufficiently precise skeleton joint tracking in many physical therapy and rehabilitation treatments requiring large "macroscopic" movements of body parts. At the same time, there are some skeleton tracking issues arising from data noise and false recognition of some scene objects. As an 
Loannis Pachoulakis and Diana Tsilidi; Technology-Assisted Carpal Tunnel Syndrome Rehabilitation using Serious Games: The Roller Ball Example. Advances in Image and Video Processing, Volume 4 No 4, August (2016); pp: 24-30

example, confusing a chair's leg with a siting patient's leg incapacitates the development of applications for individuals using wheelchairs or walkers. The results of clinical experiments with volunteers asked to perform arm and leg abductions and flexions showed that Kinect tracks movement accurately but it may underestimate arm movements and overestimate leg movements by up to $30 \%$ [13].

Techniques such as computational algorithms are also used to reduce tracking errors and improve Kinect's motion tracking accuracy. However, due to the appreciable random positional errors involved, the Kinect sensor is an appropriate therapeutic tool for wrist/hand joint tracking.

At present, the most promising candidate sensor for technology-based CTS-oriented rehabilitation seems to be the Leap Motion controller, a small USB peripheral device which is commonly placed on a table, facing upward. Using two monochromatic IR cameras and three infrared LEDs, the device commands a roughly hemispherical area out to a distance of about 1 meter from the sensor. The LEDs generate pattern-less IR light and the cameras generate frames of reflected data at a rate of close to $300 \mathrm{fps}$. This data stream is fed to the host computer through a USB cable, where Leap Motion's supporting software combines concurrent 2D frames generated by the two IR cameras to generate accurate synthetic 3D position data for the hand

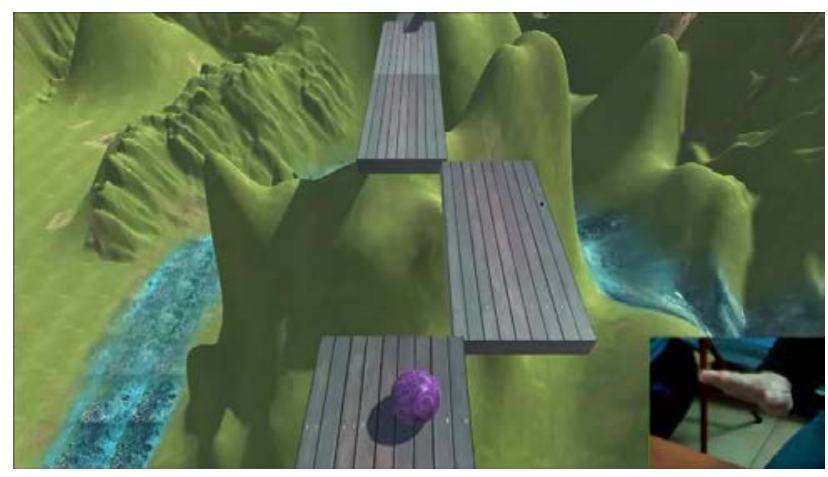

Figure 1: Sample screen-shot from the Roller Ball game tailored to Carpal Tunneling Syndrome rehabilitation. Using CTS specific exercises such as extension/flexion and radial/ulnar deviation, the patient has to successfully guide a ball on a continuously reconfigurable bridge, while avoiding a number of moving obstacles. The insert in the bottom right part of the screen-shot shows the wrist currently in an extension gesture to hold the ball back from moving ahead.

In a study conducted by [14], the Leap Motion sensor was employed in an open source (JavaScript) version of the game Fruit Ninja for stroke patient rehabilitation. The original game was modified for the needs of the study and mouse events were replaced by hand movement tracking. The pilot study involved 14 patients with stroke, who were asked to play the game for one minute, while they were able to choose the appropriate difficulty level for their condition. The results showed a general satisfaction of the users, who considered this way of rehabilitation engaging, useful and would use it at home. In this work we explore key advantages and capabilities of the Leap Motion sensor for the creation of CTS-oriented serious games. Accordingly, we have created a 3D serious game called Roller Ball - a relevant screen-shot of which appears in Figure 1 - which requires users to perform CTSspecific exercises (extensions/flexions and radial/ulnar deviations of the wrist, shown in Figure 2) to successfully guide a ball over a continuously reconfigurable bridge without falling in the void below. The bridge is made of planks, some of which periodically move to form gaps and discontinuities. In 
addition, a number of moving obstacles must be avoided to make it to the other end of the bridge without being knocked out of it.

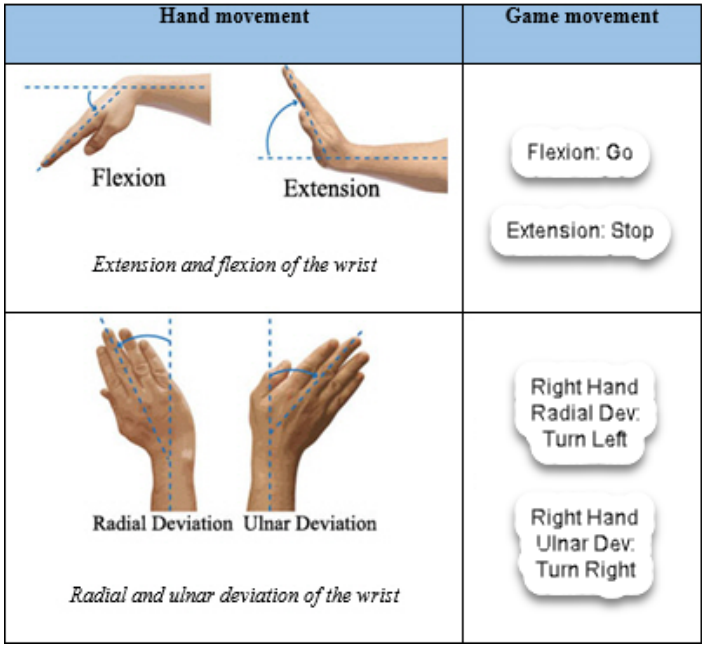

Figure 2: The left column presents four wrist physiotherapy exercises commonly used to treat Carpal Tunnel Syndrome. Each exercise is detected in the game as a gesture and is mapped onto an action on the ball as shown in the right column.

\section{Roller Ball: A Serious Game tailored to Carpal Tunnel Syndrome based on the Leap Motion Sensor}

The Roller Ball game is developed in Unity3D and uses CTS-specific physiotherapy exercises to guide a ball across a 3D scene along a bridge, while trying to avoid moving obstacles or fall off the bridge. Relevant game scenes appear in Figures 1, 3 and 4. The CTS-specific exercises used to guide of the ball appear in Figure 2 and include Flexion/Extension for the Go/Stop ball movement and Radial/Ulnar deviation to move the ball to the left/right, respectively (depending on which hand is being exercised). To make the game more challenging to the user, if one of the above gestures is not detected, the ball moves forward along a straight path at a low constant speed. Leap Motion's supporting software combines concurrent 2D frames generated by two IR cameras to generate accurate synthetic 3D position data for the hand. For each such frame, the Leap Motion API (discussed for example in [15]) uses the frame () method to expose captured motion tracking data to the application developer. This data is organized in the form of physical entities: hands, fingers and tools. A frame buffer maintains the most recent 60 frames so that a "history" parameter to the frame () method can be used to retrieve a required number frames starting from the current frame. In addition, the Frame class defines several functions that extracts useful frame data, such as a hand's position, orientation, posture, and motion:

- isRight, isLeft - parameters that identify the hand being tracked.

- Palm Position - the distance from the center of the palm to the origin of the sensor in millimeters.

- Palm Velocity - the speed and direction of movement of the palm in millimeters per second.

- Palm Normal - a vector perpendicular to the plane formed by the palm of the hand pointing downward and out of the palm.

- Direction - a vector pointing from the center of the palm toward the fingers.

- grabStrength, pinchStrength - parameters that describe the posture of the hand.

- Motion factors - a parameter group that provides relative scale, rotation, and translation factors for movement between two frames. 
Loannis Pachoulakis and Diana Tsilidi; Technology-Assisted Carpal Tunnel Syndrome Rehabilitation using Serious Games: The Roller Ball Example. Advances in Image and Video Processing, Volume 4 No 4, August (2016); pp: 24-30

Finally, the Vector class defines functions for getting the pitch (angle around the x-axis), yaw (angle around the $y$-axis), and roll (angle around the z-axis).
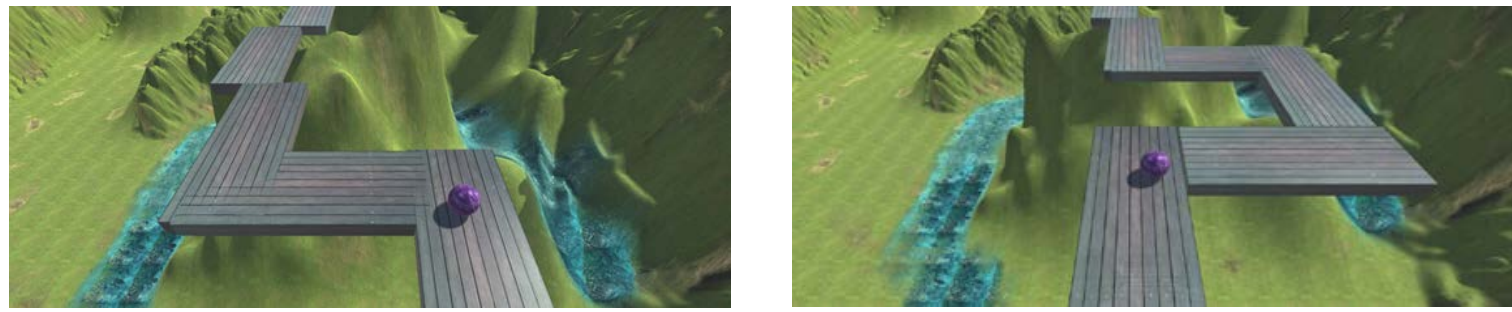

Figure 3: The top figure (a) is a screen capture showing a game scene that requires turning the ball to the left. This can be accomplished either by radial deviation of the right hand or an ulnar deviation of the left hand, depending on which hand is being exercised. Similarly, to turn the ball to the right as is required in the game scene shown in the bottom figure (b), the player must employ either an ulnar deviation of the right hand or a radial deviation of the left hand

Some customization in the response of the game to patient input is dictated by the fact that, even for a healthy hand and wrist, the angular extent for radial deviation is more limited than that for an ulnar deviation (Figure 2Figure 1). This affected the set of parameter values responsible for successful gesture detection (radial versus ulnar deviation). Further customization is naturally possible on a perpatient basis depending on the severity of the symptoms to permit exercising to the fullest range of motion that is possible to a particular patient.
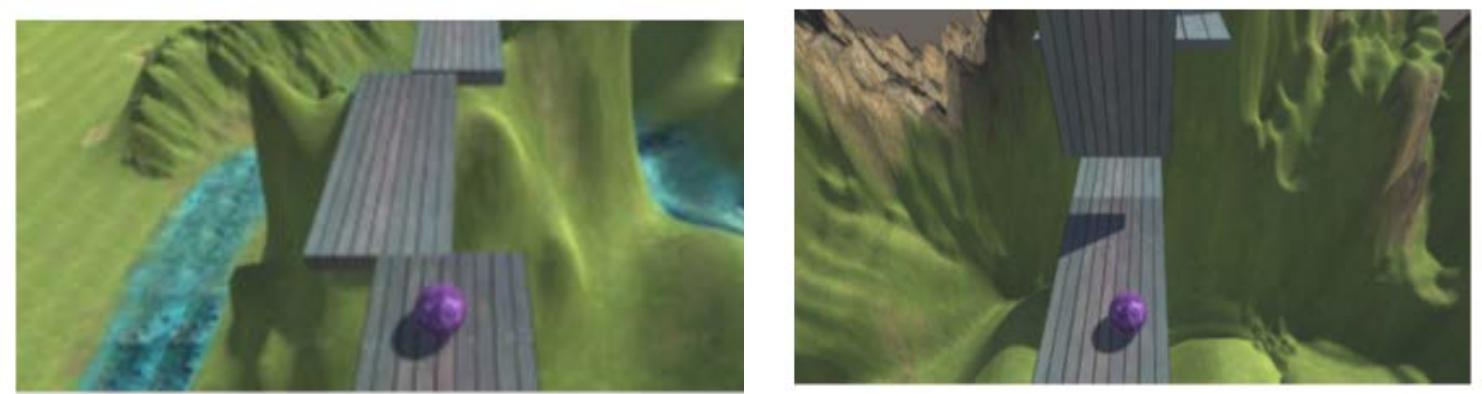

Figure 4: An obstacle in the game scene that requires wrist flexion or extension (flexion will move the ball forward, while extension will stop the ball's movement)

The game scene and the obstacles were designed to serve a game scenario that demands repetitive player actions, since in traditional physiotherapy the patient has to repeat certain movements at a specific pace. Figure 3 relates two sample game scenes. The game scene shown in Figure 3(a) requires that the player guides the ball to a left turn through either an ulnar deviation of the left hand or via a radial deviation of the right hand. By comparison, the game scene in Figure 3(b) requires that the player guides the ball to a right turn through either a radial deviation of the left hand or via an ulnar deviation of the right hand. Extension stops the movement of the ball, while flexion moves the ball forward. These wrist-hand postures can be used in game contexts such as those displayed in Figure 4. At this point it should be mentioned that the patient should use one hand for a given session so that both hands can perform the prescribed exercises correctly.

The obstacles situated in the game scene add to a more interesting and at the same time challenging game environment. Obstacles can be avoided and challenges can be surpassed by carefully timing and maintaining wrist-hand postures such as wrist flexion/extension and/or radial/ulnar deviation. At the 
same time, these obstacles urge patients to use flexion and extension of the wrist, i.e. rotation of the wrist downwards and upwards. For example, Figure 4(a) shows a board further down the bridge that constantly moves from right to left and back so that the patient must time the movement of the ball in a way that it passes over that moving plank quickly while it connects to the previous and next planks.

Another challenge appears in Figure 4(b), where a swinging vertical plank may knock the ball off the bridge. To avoid this, the patient must combine flexion and extension to time a safe passage of the ball across the obstacle. In addition, whereas in some cases the player can freely choose the movement, in other cases and depending on the form and movement of the obstacle, the player has to use specific a hand movement to avoid the particular obstacle. For example, more challenging scenarios allow for a sloped bridge where the ball develops may pick up or lose speed due to gravity. In these cases the user will be forced to perform prescribed exercises to greater deviations in a natural scenario-dependent manner.

\section{Discussion and Future Work}

In this work we investigate the potential of the Leap Motion sensor as a hardware platform on which to base serious games for the rehabilitation of patients suffering from Carpal Tunnel Syndrome (CTS). Accordingly we have presented the Roller Ball game which has been developed Unity3D to explore the possibilities and limitations of the sensor and concluded that the tracking accuracy of the sensor is sufficient to detect and track certain wrist-hand postures required by physiotherapy exercises used to alleviate CTS symptoms. Limitations in the applicability of the sensor for the intended purpose arise naturally in CTS-specific exercises where the wrist-hand posture causes part of the hand (e.g., fingers) to be physically occulted by other parts. In some of these cases the obstacles can be alleviated by removing the sensor from the default location (table top) and attaching it to a fixture that allows its re-orientation so as to view the hand from another point of view (e.g., from the top or one side).

The use of Leap Motion sensor in physical therapy and rehabilitation may appear limited, because it appropriately tracks the lower arm, hand and fingers. However this is sufficient for serious games tailored to CTS patients. In addition, the sensor can be used in conjunction with other existing platforms to be allow the calculation of additional metrics and detect and track a richer set of gestures [5]. For example, the Leap Motion sensor can be paired with Microsoft's Kinect to provide the required precision in the detection of hand gestures like pronation/supination and flexion/extension of metacarpophalangeal and the proximal interphalangeal joints of the hand, while Kinect provides larger scale body tracking. Finally, technology-enabled rehabilitation can offer measurable results as games can be designed to collect performance data and use these to calculate and store appropriate performance metrics. This possibility should allow therapists to assess long term progress and to mitigate a sense of accomplishment to the patients which can be very beneficial during treatment [16].

\section{ACKNOWLEDGEMENT}

The authors wish to thank George Stratakis, physiotherapist and manual therapy practitioner, for his input in gleaning exercises that are feasible to model using the Leap Motion Sensor.

\section{REFERENCES}

[1] R. A. Werner and M. Andary, "Carpal tunnel syndrome: pathophysiology and clinical neurophysiology," Clin. Neurophysiol., vol. 113, no. 9, pp. 1373-1381, Sep. 2002. 
Loannis Pachoulakis and Diana Tsilidi; Technology-Assisted Carpal Tunnel Syndrome Rehabilitation using Serious Games: The Roller Ball Example. Advances in Image and Video Processing, Volume 4 No 4, August (2016); pp: 24-30

[2] S. L. Michlovitz, "Conservative interventions for carpal tunnel syndrome.," J. Orthop. Sports Phys. Ther., vol. 34, no. 10, pp. 589-600, Oct. 2004.

[3] L. M. Rozmaryn, S. Dovelle, E. R. Rothman, K. Gorman, K. M. Olvey, and J. J. Bartko, "Nerve and tendon gliding exercises and the conservative management of carpal tunnel syndrome," J. Hand Ther., vol. 11, no. 3, pp. 171-179, Jul. 1998.

[4] "Physical Therapist's Guide to Carpal Tunnel Syndrome." [Online]. Available: http://www.moveforwardpt.com/symptomsconditionsdetail.aspx?cid=9f3cdf74-3f6f-40ca-b641d559302a08fc. [Accessed: 05-Mar-2016].

[5] A. Rahman, "Multisensor Serious Game-Based Therapy Environment for Hemiplegic Patients," Int. J. Distrib. Sens. Networks, vol. 2015, 2015.

[6] "Making Physical Therapy Fun with Ten Ton Raygun." [Online]. Available: http://blog.leapmotion.com/axIr8r-spotlight-making-physical-therapy-fun-with-ten-ton-raygun/.

[Accessed: 05-Mar-2016].

[7] J. C. Lee, "Hacking the Nintendo Wii Remote," IEEE Pervasive Comput., vol. 7, no. 3, pp. 39-45, Jul. 2008.

[8] F. Anderson, M. Annett, and W. F. Bischof, "Lean on Wii: Physical rehabilitation with virtua reality Wii peripherals,” Stud. Health Technol. Inform., vol. 154, pp. 229-234, 2010.

[9] G. Barry, B. Galna, and L. Rochester, "The role of exergaming in Parkinson's disease rehabilitation: a systematic review of the evidence.," J. Neuroeng. Rehabil., vol. 11, no. 1, p. 33, 2014.

[10] D. Sparks, D. Chase, and L. Coughlin, "Wii have a problem: a review of self-reported Wii relate injuries," Inform. Prim. Care, vol. 17, no. 1, pp. 55-7, 2009.

[11] M. J. D. Taylor, D. McCormick, T. Shawis, R. Impson, and M. Griffin, "Activity-promoting gaming systems in exercise and rehabilitation," J. Rehabil. Res. Dev., vol. 48, no. 10, p. 1171, 2011.

[12] H. Mousavi Hondori and M. Khademi, "A Review on Technical and Clinical Impact of Microsoft Kinect on Physical Therapy and Rehabilitation," J. Med. Eng., vol. 2014, pp. 1-16, 2014.

[13] S. Obdrzalek, G. Kurillo, F. Ofli, R. Bajcsy, E. Seto, H. Jimison, and M. Pavel, "Accuracy and robustness of Kinect pose estimation in the context of coaching of elderly population," in 2012 Annual International Conference of the IEEE Engineering in Medicine and Biology Society, 2012, pp. 1188-1193.

[14] M. Khademi, H. Mousavi Hondori, A. McKenzie, L. Dodakian, C. V. Lopes, and S. C. Cramer, "Free-hand interaction with leap motion controller for stroke rehabilitation," in Proceedings of the extended abstracts of the 32nd annual ACM conference on Human factors in computing systems - CHI EA '14, 2014, pp. 1663-1668.

[15] "Leap Motion API Reference." [Online]. Available:

https://developer.leapmotion.com/documentation/csharp/api/Leap_Classes.html?proglang=csharp.[Acc essed: 05-Mar-2016].

[16] "What if Physical Rehabilitation Were as Easy as Playing a Video Game? - Not Impossible." [Online]. Available: http://www.notimpossiblenow.com/lives/what-if-physical-rehabilitation-were-as-easyasplaying-a-video-game. [Accessed: 05-Mar-2016]. 